\title{
Risk and ranking assessments of the potential geological storage units for CCUS deployment in Portugal
}

\section{P. Pereira ${ }^{l}$, J. Carneiro $^{l}$, C. Ribeiro ${ }^{1,2}$}

${ }^{1}$ ICT - Instituto de Ciências da Terra (Universidade de Évora), ${ }^{2}$ MARE - Centro de Ciências do Mar e do Ambiente (Universidade de Lisboa)

\section{Summary}

Carbon capture, utilisation and storage (CCUS) is a key technology for the energy transition to reduce the global $\mathrm{CO}_{2}$ emissions, being crucial to mitigate emissions in hard-to-decarbonize sectors in which industrial process emission prevail. Safe storage of $\mathrm{CO}_{2}$ during a project lifetime needs to consider the potential risks of $\mathrm{CO}_{2}$ leakage from reservoirs to overburden geological formations and, ultimately, the migration pathways to the surface. Health, safety and environmental (HSE) assessments must be conducted to better understand the quality of the entire subsurface storage complexes and their suitability to deploy $\mathrm{CO}_{2}$ injection and retain it for long-term periods. This paper presents the results from the application of the HSE Screening and Ranking Framework (SRF) to the ensemble of thirtysix potential storage units located in four sedimentary basins in Portugal. The HSE SRF method enabled to improve the identification and classification of the safer storage units (and clusters) and to discard those that do not present appropriate characteristics to proceed with further detailed characterisation studies. The Lusitanian Basin, with suitable and with lower risk potential reservoirs, is the most promising area in Portugal to conduct the CCUS technology. 


\section{Introduction}

Site selection for $\mathrm{CO}_{2}$ storage in deep saline aquifers (DSA) in Portugal have identified thirty-six potential storage units (e.g. Seixas et al. 2015, Pereira et al. 2021a), grouped in eight clusters (Fig. 1) according to the distance between storage areas and the onshore/ offshore setting: two clusters in the Porto Basin (S01 and S02), three in the Lusitanian Basin (S03 and S04 located in the offshore area and the onshore cluster S05), one located in the shallow offshore of the Alentejo Basin (S06) and two in the Algarve Basin (S07 and S08).

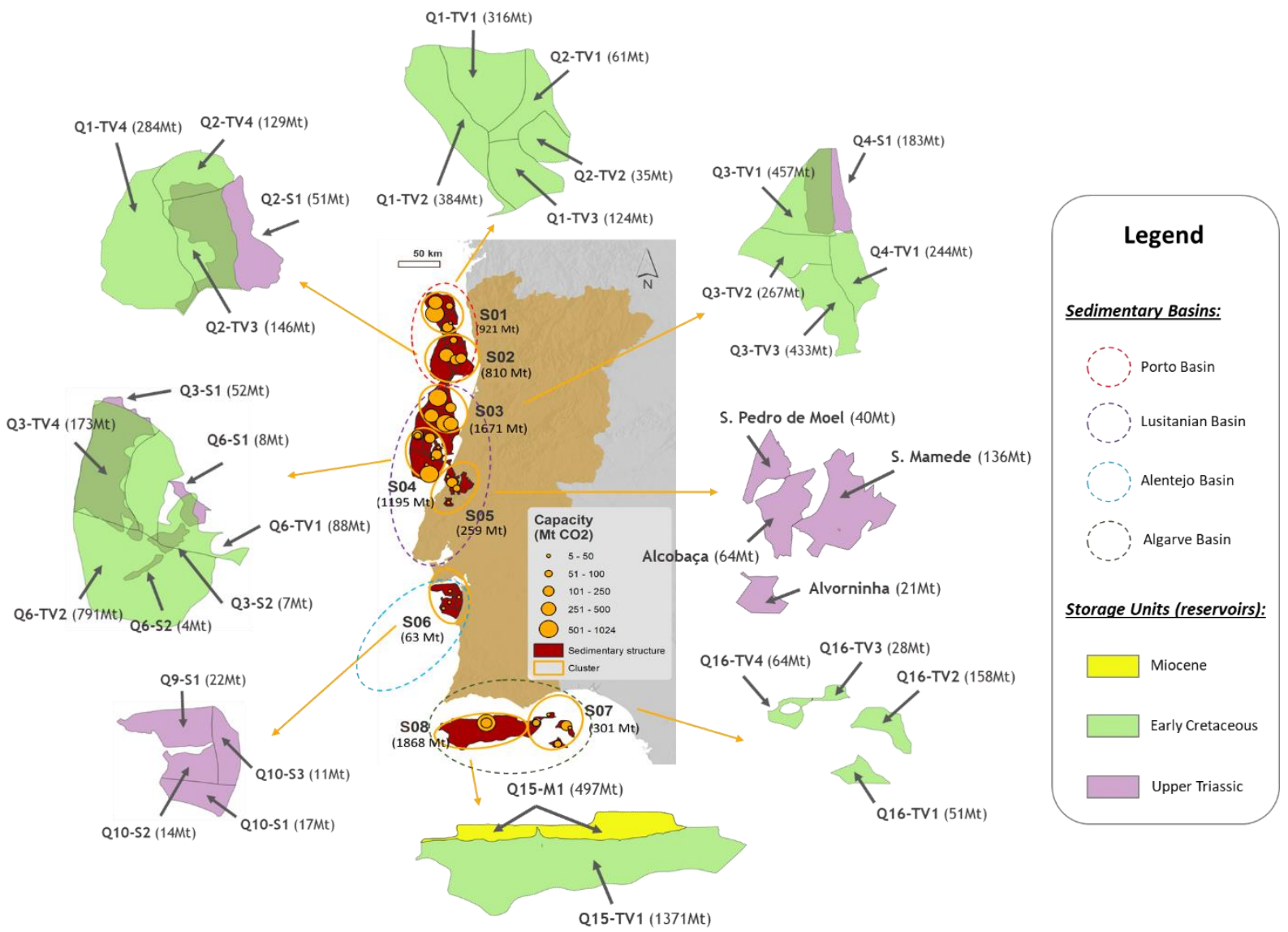

Figure 1 Location of the thirty-six storage units in Portugal and respective clusters. The individual polygons refer to the storage units in each cluster (yellow circles/ ellipses).

Geological characterisation studies performed in these sedimentary basins allowed the identification of suitable reservoir-caprock pairs: Upper Triassic siliciclastic deposits, sealed by marls, clays and evaporites from the Lower Jurassic - located both in onshore and offshore settings of the Lusitanian Basin and in the shallow offshore of the Alentejo Basin; and Early Cretaceous siliciclastic deposits, sealed by interbedded clays towards the top and by overlying Late Cretaceous limestones - located in the offshore setting of both Porto and Lusitanian basins. These are the main reservoir-caprock pairs; however, in the Algarve Basin, other promising pairs were identified namely Early Cretaceous siliciclastic sequences, sealed by Paleogene limestones, marls and clays, and a potential sandstone reservoir of Miocene age, sealed by an effective caprock of extensive Miocene-Pliocene shale deposits.

The total storage capacity of these potential reservoirs has been estimated in about $7.09 \mathrm{Gt}$, in which most of the storage potential locates in the offshore setting $(6.83 \mathrm{Gt})$, contrasting with the onshore capacity of only $260 \mathrm{Mt}$ associated with the four storage units of cluster S05 (Pereira et al. 2021a). A 
maturation assessment of the DSA resources was also conducted (Pereira et al. 2021a), resulting in two different classification levels: Tier 1 (the low-matured level, i.e., the first assessment to estimate the storage capacity of potential units) for the offshore units; and Tier 2 (the discovery assessment and the estimation of storage capacity of daughter units, i.e., suitable reservoirs) for the onshore units. Among the several sedimentary basins, the Lusitanian Basin is the most promising storage area in Portugal: the onshore Upper Triassic reservoirs are classified as Tier 2 (about $260 \mathrm{Mt}$ ), while those located in the offshore area, along with all the Early Cretaceous reservoirs, are classified as Tier 1 and with an estimated total storage capacity of about $254 \mathrm{Mt}$ and $2.6 \mathrm{Gt}$, respectively (Pereira et al. 2021b).

Injecting $\mathrm{CO}_{2}$ in geological formations is subjected to a wide variety of recognized potential pathways for leakage from deep reservoirs to the near-surface environment, (e.g., through abandoned wells or permeable fault zones) that is potentially hazardous. Nonetheless, there is also the potential for secondary entrapment, at lower depths of the storage system, and for attenuation of the $\mathrm{CO}_{2}$ plume during vertical migration (Oldenburg 2008). Unlike other risk assessment methods, the HSE SRF approach (Oldenburg 2008) allows for a quick, inexpensive and consistent framework for screening and ranking a large number of sites at early stages when characterisation data will be sparse or nonexistent. This corresponds to the Portuguese case as most of the data and knowledge available rely in the sparse direct observations and indirect geophysical data from petroleum exploration studies conducted in the country over the last decades.

\section{Methods}

The HSE SRF method, introduced by Oldenburg (2008), aims to evaluate three characteristics of a $\mathrm{CO}_{2}$ storage system (Oldenburg 2008): 1) the potential of the target formation for long-term containment of $\mathrm{CO}_{2}$ (i.e., primary containment); 2) the potential for secondary containment if the primary target leaks; and 3) the potential of the system to attenuate and/or disperse leaking $\mathrm{CO}_{2}$ if the primary formation leaks and secondary containment fails.

The workflow of the HSE SRF approach (Fig. 2) is based on a classification of three grades. In the first grade (Properties), the input values (user prior knowledge) of each assessment attribute $\left(a_{j}\right)$, the respective weight $\left(w_{j}\right)$ - that must be normalized, at the beginning of the assessment, from the sum of the weights of each attribute $(i)$, and the certainty factor $\left(c_{j}\right)$ are assigned for the set of forty-two properties $(j)$. This set of properties are grouped in nine attributes $(i)$ in the second grade (Attributes), considering, in the third grade (Characteristics), the three characteristics $(k)$ such as the primary and secondary containments and attenuation potential, obtaining the weighted assessment of attributes $\left(S_{k}\right)$ and certainty factors $\left(C_{k}\right)$. The total average attribute $\left(\operatorname{Tas}_{n}\right)$ and certainty $\left(\operatorname{Tac}_{n}\right)$, and the magnitude of total average $\left(T_{n}\right)$ are the overall scores to classify qualitatively each storage unit $(n)$ depending on the classification of parameters within each workflow grade. The input values represent proxies for site characterisation data and model analyses, that may not be available at early stages, and the acceptable intervals of values are the following: the weights $\left(w_{j}\right)$ range between 1 (least important) and 10 (most important), before normalization; the assessment of attributes $\left(a_{j}\right)$ range between -2 (poor), 0 (neutral) and 2 (excellent); and the certainty factors range between 0.1 (poorly known), 1 (generally accepted) and 2 (very well-known).

The overall classification intervals of the potential $\mathrm{CO}_{2}$ storage complexes and respective characteristics are classified as POOR, FAIR and GOOD considering the plotted areas inside and outside the POOR/GOOD quality curves (Fig. 3). Instead of discarding the original POOR/GOOD quality curves introduced by Oldenburg (2008) and replace them by the extended modifications described in Li et $a l$. 
(2013), the quality curves of both methods were considered in this work providing a wider discussion and comparison of the several results.

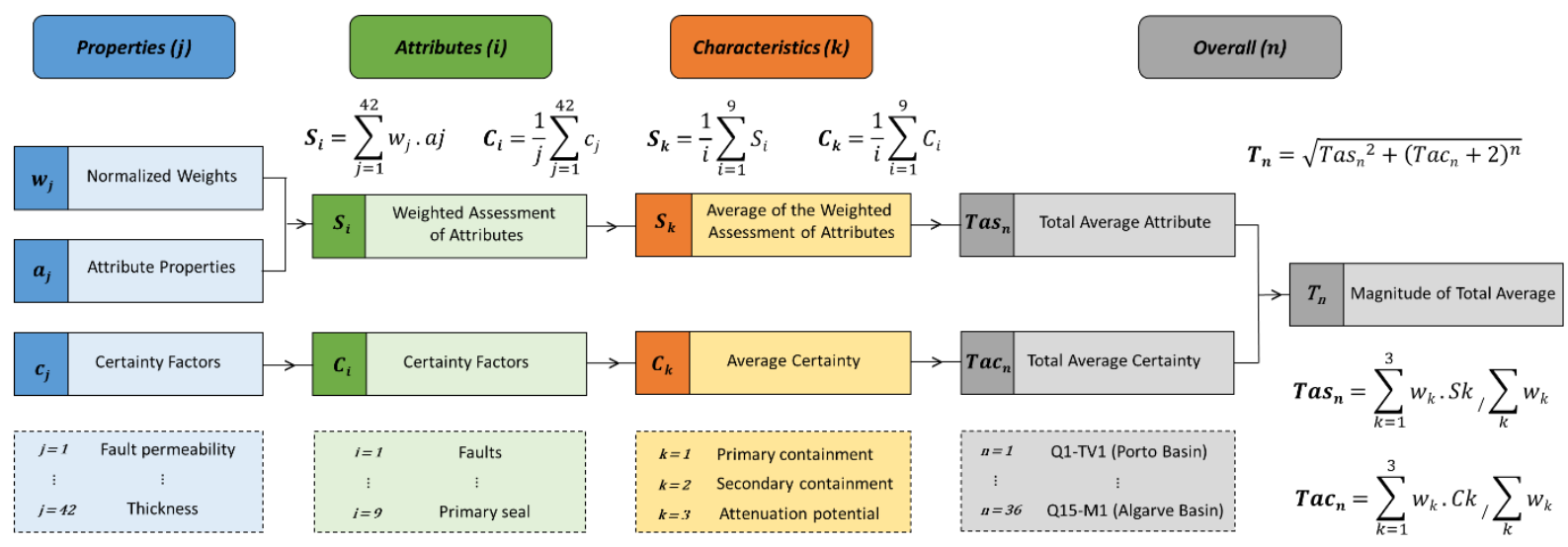

Figure 2 Workflow of the HSE SRF method (adapted from Li et al. 2013).

\section{Results}

The methodology of HSE SRF was applied to the set of storage units distributed in the sedimentary basins under study in Portugal. Figure 3 illustrates some examples resulting from different assessments. In the examples shown in Figures 3a and 3b, the storage unit Q1-TV1 (Fig. 3a) presents a classification of POOR for the secondary containment characteristic; nonetheless the value of the average for the three characteristics is quite similar (FAIR) to the storage unit Q10-S1 (Fig. 3b), which presents a classification of FAIR for the secondary containment.

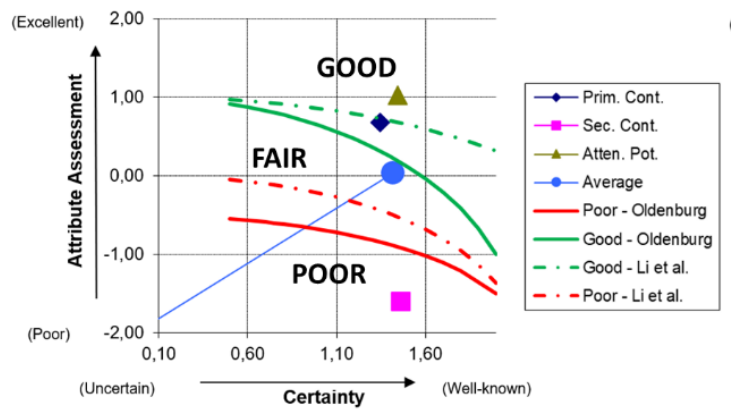

a)

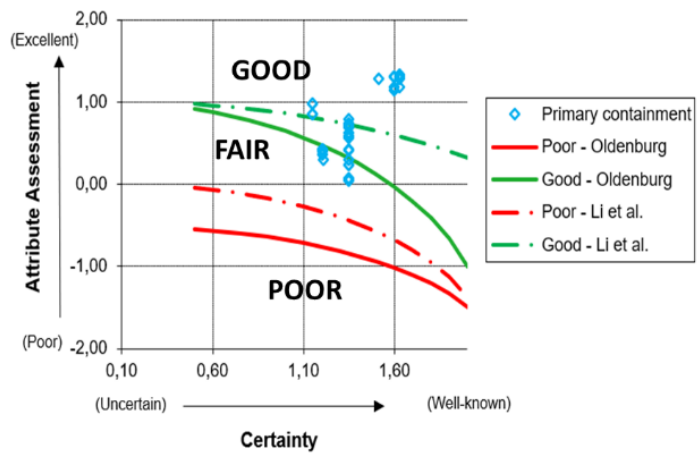

c)

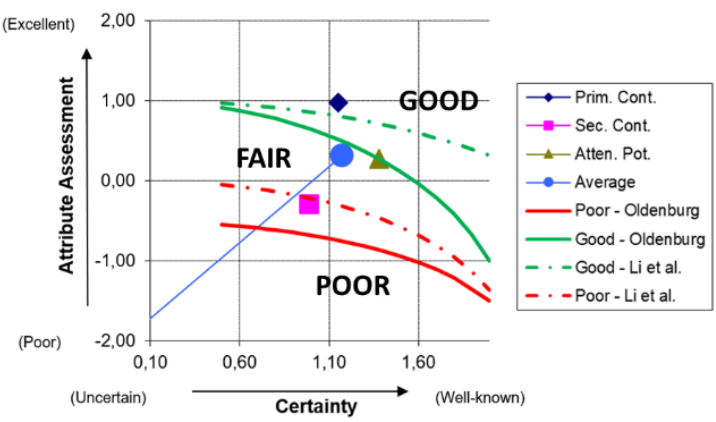

b)

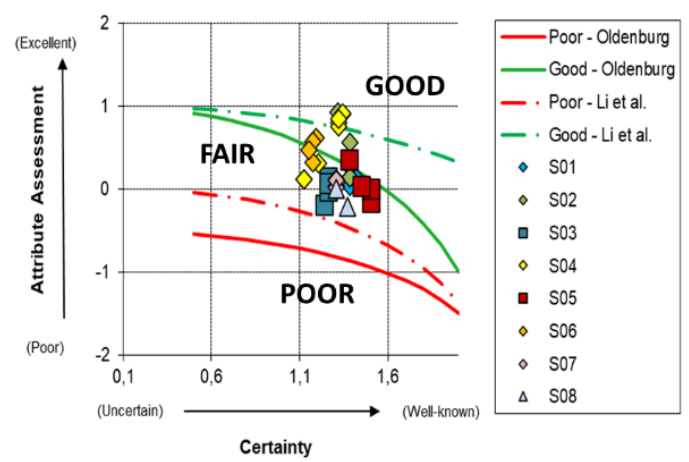

d)

Figure 3 Examples of resulting classifications of the HSE SRF method for the three characteristics of the storage unit a) Q1-TV1 (Porto Basin) and b) Q10-S1 (Alentejo Basin); c) the primary containment for the set of the thirtysix storage units; and d) the average of the three characteristics for all the storage units color-coded by the respective storage cluster. In Figures $3 \mathrm{a}$ and $3 \mathrm{~b}$, the blue segment represents the magnitude of total average $\left(T_{n}\right)$. 
Moreover, the application of both pairs of quality curves provides a broader analysis about the overall classification of each storage unit. For instance, the primary containment characteristic of most of the storage units are classified as GOOD (Figure 3c), when the Oldenburg curve is used; however, the same projected points have a different classification when the modified GOOD curve from Li et al. is applied, splitting the classification of these storage units simultaneously above and below this GOOD curve.

It is important to highlight the inexistence of storage units classified as POOR as those areas had been discarded in previous characterisation studies (the KTEJO and COMET projects, e.g., in Seixas et al. 2015). Despite the method considers some faults and tectonic properties, it lacks the integration of natural seismicity information to conduct a more realistic risk assessment, as the storage units of clusters S06, S07 and S08 present an overall classification of FAIR but they are within known areas of high seismicity risk and therefore the classifications of POOR would be expected from this assessment.

\section{Conclusions}

The HSE SRF method was applied to the set of units with $\mathrm{CO}_{2}$ storage potential in Portugal, identifying those belonging to cluster S02 (Porto Basin) and S04 (Lusitanian Basin) with lower risk of $\mathrm{CO}_{2}$ leakage and those from S03 (Lusitanian Basin) and S08 (Algarve Basin) with lower quality of the characteristics and a higher associated risk. Nonetheless, the overall assessment of the onshore potential units (S05) is classified as FAIR/GOOD. The proximity between the cluster S05 (economic advantages) and the cluster S04 (higher overall classification), reinforces the premise that this sector of the Lusitanian Basin is the most promising area for CCUS deployment, leading to the ongoing international projects STRATEGY CCUS and PilotSTRATEGY. The extension of this method to incorporate prior information of the seismicity and the assignment of neotectonics parameters to the three characteristics (and not only for the attenuation potential) would be valuable and is the way forward of this work.

\section{Acknowledgements}

This study was conducted as part of the CCS-PT project, funded by the Global CCS Institute. Participation of Pedro Pereira was in the scope of the STRATEGY CCUS project, funded by the European Union Horizon 2020 programme under grant agreement No 837754. FCT projects UIDB/04683/2020 - ICT (Institute of Earth Sciences); UIDB/04292/2020 - MARE - Marine and Environmental Sciences Centre are also acknowledged.

\section{References}

Li, Q., Liu, G., Liu, X. and Li, X. [2013] Application of a health, safety, and environmental screening and ranking framework to the Shenhua CCS project. International Journal of Greenhouse Gas Control, 17, 504-514. https://doi.org/10.1016/j.ijggc.2013.06.005.

Oldenburg, C.M. [2008] Screening and ranking framework for geologic CO2 storage site selection on the basis of health, safety, and environmental risk. Environmental Geology, 54(8), 1687-1694. 10.1007/s00254-007-0947-8.

Pereira, P., Ribeiro, C. and Carneiro, J. [2021a] Identification and characterisation of geological formations with CO2 storage potential in Portugal. Petroleum Geoscience, 27(3). 10.1144/petgeo2020-123.

Pereira, P., Carneiro, J., Ribeiro, C. and Martins J. M. [2021b] Resource maturity and sensitivity analysis of $\mathrm{CO} 2$ storage capacity in the Lusitanian basin. 82nd EAGE Conference \& Exhibition, Extended Abstract, G104.

Seixas, J., Fortes, P., Dias, L., Carneiro, J., Boavida, D., Aguiar, R., Marques, F., Fernandes, V., Helseth, J., Ciesielska, J. and Whiriskey, K. [2015] CO2 capture and storage in Portugal: a bridge to a low carbon economy. FCT-UNL, Lisbon, Portugal, 42 pp. 